\title{
Le rôle essentiel des leaders en pharmacie comme catalyseur de changement
}

\author{
par Lauza Saulnier
}

$\mathrm{E}_{\mathrm{p}}^{\mathrm{n}}$ n février 2017, j'ai participé au $27^{\mathrm{e}}$ Séminaire de gestion en macie Harrison présenté chaque année par la Société des pharmaciens d'hôpitaux (SCPH). Le programme de formation pendant cet événement offrait des occasions de perfectionnement professionnel et de réseautage destinées aux leaders en pharmacie du pays. De plus, les sources thermales d'Harrison Hot Springs me permirent d'échapper momentanément au temps froid des Maritimes.

La mise en place de nouvelles technologies, l'évolution de la pratique de la pharmacie et l'amélioration de la sécurité des soins aux patients, autant de sujets de formation qui avaient tous un point en commun : le rôle essentiel que jouent les leaders en pharmacie et les gestionnaires de pharmacie dans le succès des initiatives de changement.

De nos jours, les organismes de soins de santé doivent adopter de nouvelles technologies ainsi que des pratiques novatrices et efficientes, tout en étant ouverts au changement. Il y a plus de vingt ans, John P. Kotter, auteur et gourou du leadership, expliquait l'importance d'employer un processus par étapes afin de canaliser le changement. Il décrivait aussi les erreurs que commettent habituellement les gens cherchant à opérer un changement (Harvard Bus Rev, mai-juin 1995; www.gsbcolorado.org/uploads/ general/PreSessionReadingLeadingChange-John_Kotter.pdf). Or, les conseils de Kotter sont toujours d'actualité.

Les organismes rencontreront des difficultés en cherchant à atteindre les objectifs voulus, à moins que l'ensemble des personnes touchées par le changement vivent elles aussi leur propre transition, passant d'un état présent à un état futur. En d'autres termes, la réussite d'un changement organisationnel repose avant tout sur la participation de tous!

On enseigne aux leaders à gérer les processus et ressources de façon efficace. Or, le changement nécessite que l'on tienne compte des émotions et réactions des individus. Mais, l'aspect humain du changement et de la transition en est un avec lequel la plupart des leaders ont de la difficulté.

Plusieurs ensembles de meilleures pratiques peuvent servir à opérer un changement. Par exemple, le modèle ADKAR de la société Prosci (www.prosci.com/adkar/adkar-model) porte sur le changement à l'échelle individuelle. Il prône la mise en place des bonnes conditions pour les personnes touchées par le changement afin qu'elles adoptent de nouveaux comportements. Le sigle ADKAR désigne les cinq principes ci-dessous :
- A wareness of the need for change (conscience de la nécessité de changer);

- Desire to support and take part in the change (désir de soutenir le changement et d'y contribuer);

- Knowledge of how to change and what to do (savoir du comment et du quoi changer);

- Ability to implement the change on a day-to-day basis (capacité à mettre en ouvre le changement quotidiennement);

- Reinforcement to sustain the change (renforcement pour soutenir le changement).

La relation unique qu'entretiennent les leaders en pharmacie et les gestionnaires de pharmacie avec leurs employés fait en sorte qu'ils sont idéalement placés pour communiquer le changement, en faire la promotion, guider et influencer les employés tout au long du processus et faire face aux objections. Dans l'ouvrage Best Practices in Change Management - Édition 2016 (Prosci Inc, Fort Collins, Colorado), les participants ont indiqué que l'engagement avec les gestionnaires et les superviseurs et le soutien de ceux-ci étaient les principales conditions d'un changement réussi. Cela dit, bien que l'on reconnaisse la place cruciale qu'occupent les gestionnaires en période de changement, ils doivent d'abord être eux-mêmes favorables au changement et y être adéquatement préparés avant d'être en mesure de soutenir leurs employés.

Grâce à la SCPH, les pharmaciens d'établissements profitent d'une communauté de pratique forte et unie. Au fil des ans, j’ai appris à reconnaître la valeur des avantages "indirects " de mon adhésion à la SCPH tels que la camaraderie, l'enthousiasme à faire part d'expériences et le désir d'excellence.

Être membre actif de la SCPH vous offre beaucoup d'occasions de vous perfectionner et de mettre en pratique vos aptitudes en leadership tout en étant soutenu par des leaders en pharmacie ayant différentes expériences dans les hôpitaux et les autres milieux de soins de santé misant sur la collaboration. Profitez de cette occasion d'apprentissage unique et aidez-nous à bâtir l'avenir de la pratique de la pharmacie hospitalière en participant dès aujourd'hui aux activités de votre organisme professionnel!

[Traduction par l'éditeur]

Lauza Saulnier, B. Sc. (Pharm.), ACPR, est présidente et agente de liaison pour la vision de la Société canadienne des pharmaciens d'hôpitaux. 